\title{
EVALUATION DE L'EFFICACITE DE LA SOLUTION 8\% HUILE DE NEEM DANS LA LUTTE CONTRE LES MOUCHES TSE-TSE ET LA TRYPANOSOMOSE ANIMALE AFRICAINE AU MALI.
}

\section{THE EFFICIENCY EVALUATION OF THE NEEM OIL SOLUTION $8 \%$ IN THE FIGHTING AGAINST FLIES TSETSE AND AFRICAN ANIMAL'S TRYPANOSOMIASIS IN MALI.}

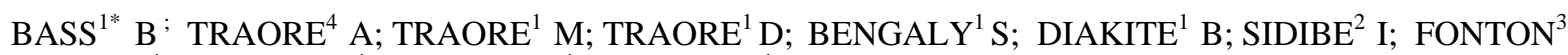 \\ $\mathrm{N}$; KONE $^{1}$ F; TRAORE ${ }^{1}$ I; SAMAKE $^{1}$ T; DIARRA ${ }^{1}$ C. O \\ 1 : Laboratoire Central Vétérinaire, Bamako, Mali. \\ 2 : Centre International de Recherche - Développement sur l'Elevage en zone Subhumide, Bobo Dioulasso, \\ Burkina Faso. \\ 3 : Université Abomey - Calavi, Benin. \\ 4 : Université des Sciences, des Techniques et des Technologies de Bamako, Mali \\ * Auteur correspondant : Boubacar BASS, entomologie, Laboratoire Central Vétérinaire. BP. 2295, Bamako, \\ Mali Tél. : + 22320243344 ; Email :drbassml1@yahoo.fr
}

\begin{abstract}
Résumé
La glossine, vecteur majeur de transmission des trypanosomoses animales et humaines est une contrainte pour le développement de l'agriculture et de l'élevage au Mali. Les activités classiques de suppression des populations de glossines s'avèrent onéreuses pour les communautés rurales pauvres ; l'utilisation anarchique et incontrôlée des trypanocides chimiques provoque des phénomènes de résistance. Il convient alors d'identifier des méthodes de lutte plus simples, peu onéreuses et plus pratiques pour les agropasteurs. L'objectif de notre étude consistait à évaluer l'efficacité de l'huile de neem contre Glossina palpalis gambiensis et à élucider les conditions de son utilisation. Pour la mise en œuvre des activités de l'étude, les responsables administratifs et socio professionnels du cercle de Dioila ont été sensibilisés. L'huile de neem utilisée pour les tests expérimentaux est une huile pure extraite des graines de neem à Tara (latitude : 1430730 ; longitude : 0308693) dans le cercle de Tominian au Mali par un groupement féminin dans le cadre de leur activité de fabrication du savon. La détermination des constituants physico - chimiques de l'huile de neem a été faite par le département médecine traditionnelle de l'Institut National de Recherche en Santé Publique du Mali. L'évaluation de l'efficacité de l'huile de neem contre Glossina palpalis gambiensis en laboratoire s'est déroulée au Centre International de Recherche - Développement sur L'Elevage en zone Subhumide (CIRDES), qui dispose d'un important élevage de Glossina palpalis gambiensis. Par semaine et pendant dix semaines, 200 glossines (100 mâles et 100 femelles) de différentes classes d'âge étaient utilisées. Différentes concentrations de la solution de l'huile de neem ont été utilisées : $2 \%, 3 \%, 4 \%, 6 \% ; 7 \% ; 8 \%$. Pour L'évaluation de l'efficacité de l'huile de neem sur Glossina palpalis gambiensis sur le terrain, Les bovins d'expérimentation (60 au total) ont subi le traitement pour « on » avec la solution $8 \%$ huile de neem (20 ml pour les animaux âgés de 3 ans et plus, $10 \mathrm{ml}$ pour ceux de moins de 3 ans). Ces animaux ne devaient pas subir d'autres traitements avec les trypanocides chimiques durant l'expérimentation (12 mois). La prévalence a été déterminée en utilisant la Technique du Buffy Coat. Les composés bioactifs présents dans l'huile de la graine de neem, la composition centésimale en acides gras de l'huile de neem et les constituants physico - chimiques de l'huile de neem ont été déterminés. Les résultats obtenus montrent que la solution $4 \%$ huile de neem a des effets insecticides et répulsifs sur Glossina palpalis gambiensis. En tenant compte d'autres facteurs du milieu (soleil, vent, pluie...) la solution $8 \%$ huile de neem préalablement testée en laboratoire a été retenue pour être utilisée sur le terrain en traitement pour « on »des bovins. La prévalence de la trypanosomose chez les bovins d'expérimentation traités à l'huile de neem était nulle. La moyenne de l'hématocrite était de 43,51.
\end{abstract}

Mots clés : Huile de neem, mouche tsé-tsé, trypanosomose animale, Mali.

\section{Summary}

The tsetse fly is the main vector for the transmission of animal's and human's trypanosomiasis; it is a constraint for the development of agriculture and livestock in Mali. The conventional activities to eliminate tsetse fly populations are expensive for the poor rural communities; the anarchic and uncontrolled use of chemical drug against trypanosomiasis cause resistance phenomena. For that it is appropriate to identify easy methods of fight wich are inexpensive and more convenient for famers. The aim of our study was to evaluate the effect of neem oil against Glossina palpalis gambiensis to clarify the conditions to use it. For the implementation of the study 
activities, administrative authorities and socio - professional of Dioila prefecture were sensitized. Neem oil used for experimental tests is a pure oil extracted from neem seeds to Tara (latitude : 1430730 ; longitude : 0308693) in Tominian in Mali by a group of women under their soap manufacturing. To determinate the physical and chemical component, the neem oil was analysis by the department of traditional medicine of National Institute for Research in Public Health of Mali. The evaluation of the effectiveness effect of neem oil against Glossina palpalis and gambiensis was made in the research center of livestock development in cool area which are breeding an important number of Glossina palpalis and gambiensis. By week since ten weeks, 200 flies (100 males and 100 females) of different age groups were used. Different concentrations of neem oil solution were used : $2 \%, 3 \%, 4 \%, 6 \% ; 7 \% ; 8 \%$ For efficiency evaluation of neem oil against Glossina palpalis and gambiensis in the field, the experimental animals (60 in total) have been treated with the solution $8 \%$ of neem oil $(20 \mathrm{ml}$ for animals more than 3 years old and $10 \mathrm{ml}$ for animals under 3 years old). Those animals should not be treated by other chemical drugs during the experimentation (12 months). Bioactive compounds in the seed of neem oil, the percentage of fatty acid in the composition of neem oil, the physical and chemical components of neem oil were determined. The results show that the solution $4 \%$ of neem oil has insecticidal and repellent effects on Glossina palpalis and gambiensis. Taking into account other factors in the field (sun, wind, rain ...) the solution $8 \%$ neem oil previously tested in the laboratory was chosen to be used on the field for the treatment "on" cattle. Any trypanosome parasite has been found in experimental cattle treated with neem oil. The mean hematocrit was 43,51 .

Keywords: Neem oil, tsetse fly, animal's trypanosomiasis, Mali.

\section{Introduction}

Le neem ou Azadirachta indica est un arbre de la famille des Méliaceae, originaire d'Inde. Il est peut être la plus utilisée des plantes médicinales en Inde (1). Le neem est un arbre sauvage dont la croissance est rapide et la durée de vie très longue puisqu'il peut vivre plus de deux siècles. C'est un grand arbre, aux branches longues et étalées, qui peut atteindre 30 mètres de haut. Son système radiculaire s'étend de façon importante en largeur et en profondeur. Ses feuilles sont légèrement dentelées, pennées, et de couleur verte foncée. Les inflorescences apparaissent à la fin du printemps. De couleur blanche, elles développent un parfum sucré. Le fruit est une drupe ovale entourée d'une pulpe charnue de couleur jaune pâle, à haute teneur en huile. Le neem pousse de préférence dans les régions tropicales et subtropicales. Cependant, c'est un arbre peu exigeant qui s'adapte facilement aux conditions pédoclimatiques de son environnement. Il supporte des températures allant de $4^{\circ} \mathrm{C}$ à $44^{\circ} \mathrm{C}$ et une pluviométrie variant entre $400 \mathrm{~mm}$ et 1500 $\mathrm{mm}$. Il peut pousser jusqu'à une altitude de 1500 mètres. Sa distribution géographique est donc très étendue puisqu'il est présent en Inde, son milieu d'origine, en Indonésie, en Australie, en Afrique, dans le bassin Méditerranéen, en Amérique Latine et au sud des Etats-Unis. De nombreuses études ont été menées dans plusieurs pays et aux Etats-Unis en particulier sur la possible toxicité du neem. Ces études ont montré que les feuilles et l'écorce de neem ont une très faible toxicité (2-5). La teneur en huile de la graine de neem varie de 40 à $50 \%$ et l'extraction permet d'obtenir environ $150 \mathrm{ml}$ d'huile à partir d' un $\mathrm{Kg}$ de poudre de graine. $\mathrm{La}$ glossine, vecteur majeur de transmission des trypanosomoses animales et humaines est une contrainte pour le développement de l'agriculture et de l'élevage au Mali. Les activités de suppression des populations de glossines par l'installation de pièges et d'écrans imprégnés et le traitement épi cutané des animaux domestiques par des insecticides synthétiques importés et très onéreux, sont à la charge des communautés rurales. Le projet vise par conséquent à élucider les conditions d'utilisation et à évaluer l'efficacité de l'huile de neem contre Glossina palpalis gambiensis afin de mieux lutter contre les trypanosomoses et contribuer aux efforts déployés par les communautés rurales pour améliorer leur situation économique et sanitaire. Les formulations chimiques en dépôt dorsal diffusent rapidement sur la plus grande partie du corps et se mélangent aux secrétions cutanées. Bien tolérées et presque sans risque de résidus ou de danger, elles permettent de s'affranchir des installations lourdes et onéreuses. Cette méthode est très prisée des éleveurs qui préfèrent effectuer une manipulation sur leur bétail plutôt que sur un piège (7). C'est la méthode la plus utilisée au Mali. Toute fois, ces formulations s'avèrent onéreuses, or en santé vétérinaire, la rentabilité économique est essentielle; il convient dès lors de chercher les méthodes de lutte plus simples, peu onéreuses et plus pratiques pour les agropasteurs. Les résultats obtenus sur les glossines par contact tarsal ne permettent pas d'envisager l'utilisation des extraits de neem en pulvérisation directe ou par le traitement résiduel des lieux de repos des glossines ainsi que leur emploi pour imprégnation de leurres (8). L'effet répulsif risque en outre de réduire le temps de contact de l'insecte avec le produit. L'étude réalisée avec 
l'azadirachtine a également montré une forte activité larvicide, pupicide et adulticide de l'azadirachtine sur Anopheles stephensi, vecteur du paludisme. (9)

L'azadirachtine, est le composé le plus actif contre les insectes (400 espèces d'arthropodes nuisibles). Il agit à la fois sur la croissance et le développement de l'insecte (croissance larvaire, mue), et comme facteur anti nutritif. (10). La question de recherche à laquelle nous avons tenté de répondre était la suivante : la solution $8 \%$ huile de neem est - elle efficace dans la lutte contre les glossines et les trypanosomoses animales africaines?

\section{Méthodologie}

Pour la mise en œuvre de l'étude la stratégie adoptée comprend les phases suivantes :

- La sensibilisation;

- L'extraction de l'huile de neem ;

- La détermination des constituants physico - chimiques de l'huile de neem ;

- L'évaluation de l'efficacité de l'huile de neem sur Glossina palpalis gambiensis en laboratoire ;

- L'évaluation de l'efficacité de l'huile de neem sur les trypanosomes en milieu naturel.

\subsection{Sensibilisation :}

Une mission du responsable de l'étude s'est rendue à Dioïla et Koulikoro pour rencontrer les responsables de la chambre régionale d'agriculture de Koulikoro, les responsables de la chambre locale d'agriculture de Dioilla, les membres de la commission régionale des utilisateurs de Koulikoro, les membres de l'association locale des éleveurs de Dioïla, le service vétérinaire de Dioïla. Des informations relatives à l'objectif et à la méthodologie de l'étude (choix de deux villages, Dioïla et Ténébougou) ont été données.

\subsection{Huile de neem}

L'huile de neem utilisée pour les tests expérimentaux est une huile pure extraite à Tara (latitude : 1430730 ; longitude : 0308693) dans le cercle de Tominian au Mali par un groupement féminin dans le cadre de leur activité de fabrication du savon. Comme dans le cas de toutes les graines oléagineuses, l'huile de la graine de neem est localisée dans les cellules de l'amande sous forme de globules lipidiques. Son extraction est faite par une action mécanique de pressage libérant les gouttelettes d'huiles présentes dans ces cellules. Les graines sont au préalable décortiquées et vannées pour éliminer les débris des envéloppes. Elles sont ensuite broyées et transformées en une pâte qui est malaxée pour en extraire l'huile de couleur marron presque opaque et d'une odeur épicée bien spécifique.

\subsection{Détermination des constituants physico -} chimiques de l'huile de neem:

La détermination des constituants physico chimiques de l'huile de neem a été faite à l'université d'Abomey - Calavi. Par exemple, la détermination de la teneur en azadirachtine dans les solides s'est faite en trois étapes : l'extraction, la purification et l'analyse. L'analyse est effectuée en Chromatographie Liquide haute Performance (CHLP) grâce à un appareil HPLC ASI 100 muni d'une colonne C18 (250 x 4,6 mm OMNISPHCR 5 C18) et d'un détecteur UV visible.

\subsection{Evaluation de l'efficacité de l'huile de neem} sur Glossina palpalis gambiensis en laboratoire:

Cette étude a été faite au Centre International de Recherche - Développement sur L'Elevage en zone Subhumide (CIRDES), qui dispose d'un important élevage de Glossina palpalis gambiensis.

Les individus sont maintenus dans des cages de type « Roubaud» à raison de 25 et 50 individus par cage. Deux cent glossines (100 mâles et 100 femelles) de différentes classes d'âge étaient utilisées par semaine pendant dix semaines. Différentes concentrations de la solution de l'huile de neem (huile de neem plus eau distillée) ont été utilisées : 2\%, 3\%, 4\%, 6\%;7\%;8\%. Différentes manipulations ont été effectuées :

- Applications de la solution de l'huile de neem sur la face dorsale du thorax :

$2 \mu 1$ de solution étaient appliqués sur la face dorsale du thorax de chaque mouche.

Les glossines étaient traitées individuellement par micropipette. Avant chaque manipulation, elles étaient nourries puis anesthésiées dans la salle de test. Pour évaluer l'effet de l'huile de neem sur la mortalité, les tests étaient effectués avec des femelles âgées et gravides ainsi que des mâles de plus de dix jours.

Les glossines mortes ou affaiblies étaient comptées et retirées des cages. Les tests étaient effectués sur de jeunes femelles pour suivre l'impact de traitement sur la reproduction. Les femelles de 2-3 jours étaient accouplées avec des mâles de 7-10 j. Elles étaient mises en présence des mâles pendant 72 jours, puis les sexes étaient séparés.

Chaque jour, les pupes étaient récoltées et comptées. Le nombre d'imagos émergent était compté pour déterminer le taux d'éclosion.

- Contact forcé de Glossina palpalis gambiensis sur du tissu bleu imprégné :

Du tissu bleu (identique à celui rentrant dans la fabrication des pièges utilisés dans les campagnes de lutte) est imprégné à différentes concentrations $(8 \% ; 7 \% ; 6 \% ; 4 \% ; 3 \% ; 2 \%)$ d'huile de neem. 
Les mouches non anesthésiées étaient mises en contact avec le tissu traité par les tarses en les maintenant par les ailes pendant 15,30 et 60 secondes. Le témoin était constitué par un lot de mouches manipulées de la même façon, mais sur du tissu non traité. Les tests étaient effectués sur des femelles âgées et gravides ainsi que sur des mâles de plus de dix jours. Les paramètres de suivi étaient identiques pour tous les lots de glossines.

- Contact spontané de Glossina palpalis gambiensis sur les flancs des animaux (vache et chèvre) :

Des glossines (femelles âgées et gravides, jeunes femelles, jeunes mâles) à jeun depuis 72 heures et mises par lots de 25 en cage, étaient nourries sur un flanc des hôtes nourriciers dont la peau est badigeonnée à différentes concentrations $(8 \% ; 7 \%$; $6 \% ; 4 \% ; 3 \% ; 2 \%$ ) de la solution d'huile de neem. La cage témoin était posée sur le flanc non traité. Les cages étaient maintenues $10 \mathrm{mn}$ sur l'hôte nourricier. Le nombre de glossines qui prenait un repas de sang était compté.

Evaluation de l'efficacité de l'huile de neem sur les trypanosomes en milieu réel:

L'étude eût lieu de janvier 2011 à juillet 2012 dans deux localités du cercle de Dioïla : la commune de Dioïla et le village de Ténébougou. Ces deux localités avaient la même Densité Apparente de la Population de glossines et le même taux de prévalence de la trypanosomose (BASS et al, 2010).

Les animaux d'expérimentation subissaient le traitement pour « on » avec la solution $8 \%$ huile de neem $(20 \mathrm{ml}$ pour les plus de 3 ans et $10 \mathrm{ml}$ pour les moins de 3 ans).

Les positions géographiques des troupeaux ont été relevées à l'aide du GPS.

Les autres animaux subissent le traitement avec les trypanocides chimiques classiques.

Les animaux ont été divisés en deux lots en fonction de la fréquence du traitement avec la solution $8 \%$ huile de neem :

Lot 1 : traitement mensuel

Lot 2 : traitement bimensuel

Les animaux ayant participé à l'étude ont été fournis par l'association des éleveurs et la chambre locale d'Agriculture. Ainsi, trois troupeaux de bovins ont été choisis à Dioïla village et deux troupeaux à Ténébougou.

Au total soixante (60) bovins mâles et femelles de différentes classes d'âge ont été choisis avec l'accord des propriétaires d'animaux. Ces soixante bovins ont été traités avec la solution $8 \%$ huile de neem seulement.

Dioïla village :

\begin{tabular}{|c|c|c|c|}
\hline $\begin{array}{l}\text { Troupeau I } \\
\text { d'expérimentation. }\end{array}$ & (BSIM) : & 13 & animaux \\
\hline $\begin{array}{l}\text { Troupeau II } \\
\text { d'expérimentation. }\end{array}$ & (ATRA) : & 12 & animaux \\
\hline $\begin{array}{l}\text { Troupeau III } \\
\text { d'expérimentation. } \\
\text { Ténébougou : }\end{array}$ & (FMAN) : & 10 & animaux \\
\hline $\begin{array}{l}\text { Troupeau I } \\
\text { d'expérimentation. }\end{array}$ & $(\mathrm{NCOU}):$ & 13 & animaux \\
\hline $\begin{array}{l}\text { Troupeau II } \\
\text { d'expérimentation. }\end{array}$ & (STRA) : & 12 & animaux \\
\hline
\end{tabular}

La recherche des trypanosomes chez les animaux retenus pour l'étude a permis de déterminer la prévalence de la maladie. Pour cela, le sang prélevé dans des tubes «vacutainer» avec anticoagulant était aussitôt analysé en utilisant la Technique du Buffy Coat : centrifugation, lecture de l'hématocrite et examen en microscopie en fond noir.

\section{Résultats}

\subsection{Caractéristiques physico-chimiques de l'huile de neem:}

Aspect : pâteux à $20^{\circ} \mathrm{c}$

Odeur : épicée caractéristique

Couleur : vert marron

Densité : 0.908 à 0.934 à $30^{\circ} \mathrm{c}$

Indice de saponification : 175 - 205

Indice d'Iode : $65-80$

Indice de réfraction : $1.4615-1.4705$ à $40^{\circ} \mathrm{C}$

Autres : thermolabile et photodégradable

Les composés bioactifs présents dans l'huile de la graine de neem : Nimbin, Nimbolide, Gédunine, Azadirachtine, Mahmoodine. L'azadirachtine est le composé le plus actif contre les insectes.

La composition centésimale des différents acides gras présents dans l'huile de neem est donnée dans le tableau 1

\subsection{Evaluation de l'efficacité de l'huile de neem sur Glossina palpalis gambiensis en laboratoire}

- Dans le cas de l'application de la solution de l'huile de neem à différentes concentrations (2\%-8\%) sur la face dorsale de Glossina palpalis gambiensis (Tableau 2), il ressort que quels que soient la classe d'âge, le sexe ou l'état physiologique de la glossine, les taux de mortalité variaient entre $0-2 \%$ pour une concentration de $2 \%$ d'huile de neem à $100 \%$ pour les concentrations allant de $4 \%$ à $8 \%$.

- Dans le cas d'un contact forcé de Glossina palpalis gambiensis sur du tissu bleu imprégné (Tableau 3), La corrélation dose - mortalité est confirmée sur du tissu bleu imprégné avec différentes concentrations 
de l'huile de neem. Les taux de mortalité étaient de $0 \%$ à $2 \%$ de concentration d'huile, $50 \%$ pour une concentration de $6 \%$ et $100 \%$ de mortalité pour des concentrations allant de 7-8\% d'huile quel que soit le sexe:

Dans les deux cas (applications topiques et tissus imprégnés) la mortalité était élevée au fur et à mesure que les concentrations de l'huile de neem augmentent.

- Glossina palpalis gambiensis est soumise à un contact forcé avec du tissu bleu non imprégné, aucun Cas de mortalité n'était observé quel que soit le temps de contact.

- Quand Glossina palpalis gambiensis est mis en contact spontané sur les flancs des animaux, le nombre de glossines gorgées diminuait proportionnellement à la concentration de l'huile de neem badigeonnée sur le flanc de l'animal. Les résultats sont mentionnés dans le tableau 4. Il ressort de l'analyse du tableau que les solutions $6 \%, 7 \%$ et $8 \%$ huile de neem ont à la fois des effets insecticides et répulsifs sur Glossina palpalis gambiens en laboratoire.

\subsection{Evaluation de l'efficacité de l'huile de neem en traitement pour « on » sur les bovins}

Nous avons présenté les différents résultats obtenus dans les tableaux 5, 6 et 7. IL en ressort que l'action de l'huile de neem sur les parasites s'est traduite par une prévalence nulle quelle que soit l'espèce de trypanosome (Tableau 5). La moyenne de l'hématocrite était comparable que les animaux soient traités mensuellement $(44,2)$ ou bimensuellement $(43,2)$ avec la solution $8 \%$ de l'huile de neem (Tableau 6). Il apparait cependant que la moyenne de l'hématocrite soit plus faible, 34,6 chez les bovins traités avec les trypanocides classiques (Tableau7).

\section{Discussion}

Nous avons obtenu 28\% de rendements d'extraction en huile brute. Ces résultats étaient comparables à ceux obtenus en Inde (27\%) (11), mais légèrement inférieurs à ceux obtenus en Côte d'Ivoire (30\%) (12). Le procédé d'extraction par pression mécanique à froid que nous avons utilisé pour produire l'huile permettait d'éviter toute altération de l'huile. Cette qualité d'huile était meilleure à celle obtenue par Koul et al. 1990, selon lesquels l'huile de neem produite par une action mécanique de pressage était de moins bonne qualité. Nos résultats étaient différents de ceux obtenus au Sénégal (14) pour certains composants dont l'Acide Oléique $=42.97 \%$; Acide Linoléique $=0.50 \%$; Acide Palmitique $=17.65 \%$. Cette différence peut s'expliquer par l'extraction au cyclohexane des graines du Sénégal. Les fortes mortalités obtenues chez les femelles âgées et gravides et sur les jeunes mâles lors des applications topiques, traduisaient une relation dose/mortalité plus forte que celle généralement observée avec les insecticides conventionnels de synthèse. Toutefois, de faibles taux de mortalité ont été plutôt observés avec l'utilisation de fortes concentrations d'une préparation commerciale contenant de l'azadirachtine (Azantin EC) chez les femelles gravides et les jeunes mâles de Glossina fuscipes fuscipes (8). A l'opposé des taux élevés de mortalité que nous avons obtenus avec l'utilisation du tissu imprégné à l'huile de neem, de faibles taux de mortalité ont été enregistrés ailleurs sur Glossina fuscipes fuscipes en utilisant une formulation de l'azadirachtine [8]. La différence entre nos résultats parce qu'une formulation commerciale de l'azadirachtine était utilisée sur Glossina fuscipes fuscipes, au lieu de l'huile pure. La forte mortalité observée par contact tarsal permet d'envisager l'utilisation de la solution $8 \%$ huile de neem dans la lutte antiglossines par écrans ou pièges imprégnés. La baisse significative du poids des pupes à partir de la solution $4 \%$ huile de neem que nous avons observée était confirmée par d'autres auteurs $(8,15)$ qui avaient nourri des femelles de Glossina palpalis palpalis sur les flancs de chèvre traités avec des extraits de feuilles de neem. Il apparait donc que le traitement avec l'huile de neem affecte la productivité et le poids des pupes. Des résultats similaires ont été trouvés sur Locusta migratoria après injection de l'azadirachtine (16). Par extrapolation, on peut donc affirmer qu'à partir de la solution $4 \%$ huile de neem, la sécrétion d'hormones est affectée chez l'adulte de Glossina palpalis gambiensis et les taux d'éclosion sont réduits fortement. A ces travaux également ceux ayant conduit à des inhibitions d'émergence d'adultes chez Musca autumnalis après une exposition des larves à l'azadirachtine (17). En comparant deux préparations commerciales (Margosan-O et Azantin EC), certains auteurs ont montré que des composants de l'huile de neem augmentent significativement l'effet insecticide des préparations commerciales sur Acyrthosiphon pisum (Stark et Walter, 1995) [18]. Après avoir enduit l'oreille de lapin avec la formulation d'azadirachtine, Makoundou et al. (1995) ont observé qu'une proportion très importante de glossines à jeun refusait de se nourrir. Nous avons 
obtenu un résultat similaire en appliquant différentes concentrations de la solution d'huile de neem sur les flancs des vaches et chèvres. Avec les solutions $7 \%$ et $8 \%$ huile de neem, $100 \%$ des glossines ont refusé de s'alimenter après 72 heures de jeûne. Contrairement à nos résultats, D. Dankwa (non publié) en badigeonnant les flancs de chèvre avec des extraits de feuilles de neem, aucun effet répulsif n'a été observé. Le pourcentage d'individus dont l'hématocrite était inférieur à $25 \%$ (seuil classiquement considéré comme révélateur d'un état pathologique) était de 5\% (3/60) chez les animaux traités avec la solution $8 \%$ huile de neem, et de $18 \%(35 / 190)$ chez les animaux traités avec les trypanocides classiques. L'hématocrite apparait ainsi comme un indicateur pertinent de l'infection trypanosomienne active des bovins, en accord avec les résultats d'autres auteurs $(19,20)$. Outre son action sur les vecteurs biologiques intervenant dans la transmission, la solution $8 \%$ huile de neem agit également sur les vecteurs mécaniques notamment les Tabanidés et les Stomoxes qui assurent la transmission en relais de Trypanosoma vivax. L'effet répulsif de la solution $8 \%$ huile de neem en application cutanée laisse entrevoir quelques perspectives dans le domaine de l'élevage en Afrique, notamment en assurant la protection du bétail contre les piqûres de glossines. Il est connu que dans certaines situations (transhumance du bétail), I'application d'un produit répulsif peut avoir un intérêt en empêchant ou en limitant le contact glossines infectées-bétail, réduisant ainsi la transmission des trypanosomoses. La solution $8 \%$ huile de neem de par son effet répulsif pourra constituer un moyen simple de protéger localement les animaux (un arbre fournit $30 \mathrm{~kg}$ de graines donnant de 6 à $8 \mathrm{~kg}$ d'huile) et un moyen de lutte gérable par les communautés rurales.

\section{Conclusion}

L'objectif de notre étude était d'évaluer l'efficacité et les conditions d'utilisation de l'huile de neem contre les glossines et les trypanosomes. Les taux élevés de mortalité observés par contact tarsal permettent d'envisager l'utilisation de la solution $8 \%$ huile de neem en pulvérisation directe ou par le traitement résiduel des lieux de repos des glossines (troncs et branches d'arbres) ainsi que leur emploi pour imprégnation de leurres (pièges et écrans). Les effets sur la mortalité et sur la reproduction lors d'applications topiques donnent en outre l'espoir d'un usage sur le terrain. Dans le cadre d'une application sur le terrain en vue de mettre à profit l'effet répulsif, d'autres études seraient cependant utiles à effectuer. Il s'agit notamment de la rémanence sur le pelage, le coût de l'utilisation, la toxicité pour le bétail, les résidus dans le lait et la viande.

\section{Références}

1. Biswas K, Chattopadhyay I, Banerjee R.K, Bandyopahyay U. 2002: Biological activities and medicinal properties of neem (Azadirachta indica). Current Science, 82, 1336 - 1345.

2. Chattopadhyay R.R, Sarkar S.K, Ganguly S, Banerjee R.N. 1992: Active effects of Azadirachta Science and Culture, 58: 39 - 40.

3. Challier, 1984: Le piégeage des tsé-tsé. Maisons - Alfort, France, Cirad - emvt, 172P

4. Grebault Pascal. UMR177 IRD/CIRAD ; Montpellier 2009-2010 : Glossine et transmission des

trypanosomoses africaines.http://www.mpl.ird.fr/ur 016/file/09\%20Module\%20entomo\%202010\%20$\% 20$ Grebault $\% 20-$

\%20Glossines\%20et\%20THA.pdf consulté le 2203-2015

5. Cuisance D, Boutras, 1995 : Effet sur Glossina palpalis gambiensis de substances médicamenteuses administrées au lapin, hôte nourricier. Revue Elev. Med. Vet pays tropicaux (32) : 37-46

6. Nathan S; Kalaivani K, 2005: Effects of neem limonoids on the malaria vector Anopheles stephensi Liston (Diptera: Culicidae). Acta Tropica 96 (2005) $45-55$.

7. www.neem.fr , consulté le 18/03/2015

8. Khattak S.G, Gilani S.N, Ikram M. 1985: Antipyretic studies on some indigenous Pakistani medical plants. Journal of Ethnopharmacology, 14: $45-51$.

9.Kaura, S.K; Gupta, S.K; Chowdhury, J.B, 1998 : Morphological and oil content variation in seeds of Azadirachta indica A. Juss (Neem) from northern and western provenances of India plants Foods for Human Nutrition, 52, 293 - 298.

10. Gosse, B; Amissa, A.A; Adje, F.N; Niamke, F.B; 1992: Analysis of components of neem (Azadirachta indica) oil by diverse chromatographic techniques. Journal of Liquid Chromatography and Related Technologies, 28, 2225 - 2233.

11. Laveissière et al, 1980 : Un nouveau piège pour la capture des glossines (Glossine : diptera, Glossinidae). Revue Elev. Med. Vet. Pays tropicaux ; $37: 31-59$

12. Koul O, Isman M.B, Ketkar C.M.1990: Properties and uses of neem, Azadirachta indica. Canadian Journal of Botany, 68, 1-11.

13. Mamadou FAYE, 2010. Thèse de Doctorat de l'université de Toulouse. « Nouveau procédé de fractionnement de la graine de neem (Azadirachta 
indica A. juss) sénégalais : production d'un Bio pesticide d'huile et de tourteau »

14. Sami BARREK, Olivier PAISSE, MarieFlorence GRENIER, 2010 : Service Central d'Analyse - USR 059 - CNRS.

15. Makoundou P.B. et $a l, 1995$ : Etude au laboratoire des effets d'un insecticide naturel extrait du neem (Azadirachta indica A. juss) sur Glossina fuscipes fuscipes Newstead, 1910 (Diptera:

Glossinidae). Revue Elev. Med. Vet. Pays trop. ; 48 (4) : 339-345

16. Rembold et Coll., 1987: Effect of azadirachtin A on hormone titers during the gonadotrophic cycle of Locusta migratoria. Eschbom, Allemagne, GTZ, p.289-298
17. Gaaboub I.A, Hayes D.K, 1984: Biological activity of azadirachtin, component of the neem tree inhibiting molting in the face fly, Musca autumnalis. Environ. Entomol. 13: 803-812 18. Stark J.D, Walter J.F, 1995: Neem oil components affect the efficacity of commercial neem insecticides. J; Agric. Food chem., 43: 507512

19. Saraf A.P, Joglekan V.K. 1993: Study of effect of "karnim" in patients of non insulin dependent diabetes mellitus.

20. Simiah D and Baskaran G. 1981: Margosa oil poisoning as a cause of Reye's syndrome. Lancet, 1, $487-489$.

\section{$\underline{\text { Tableaux et figures }}$}

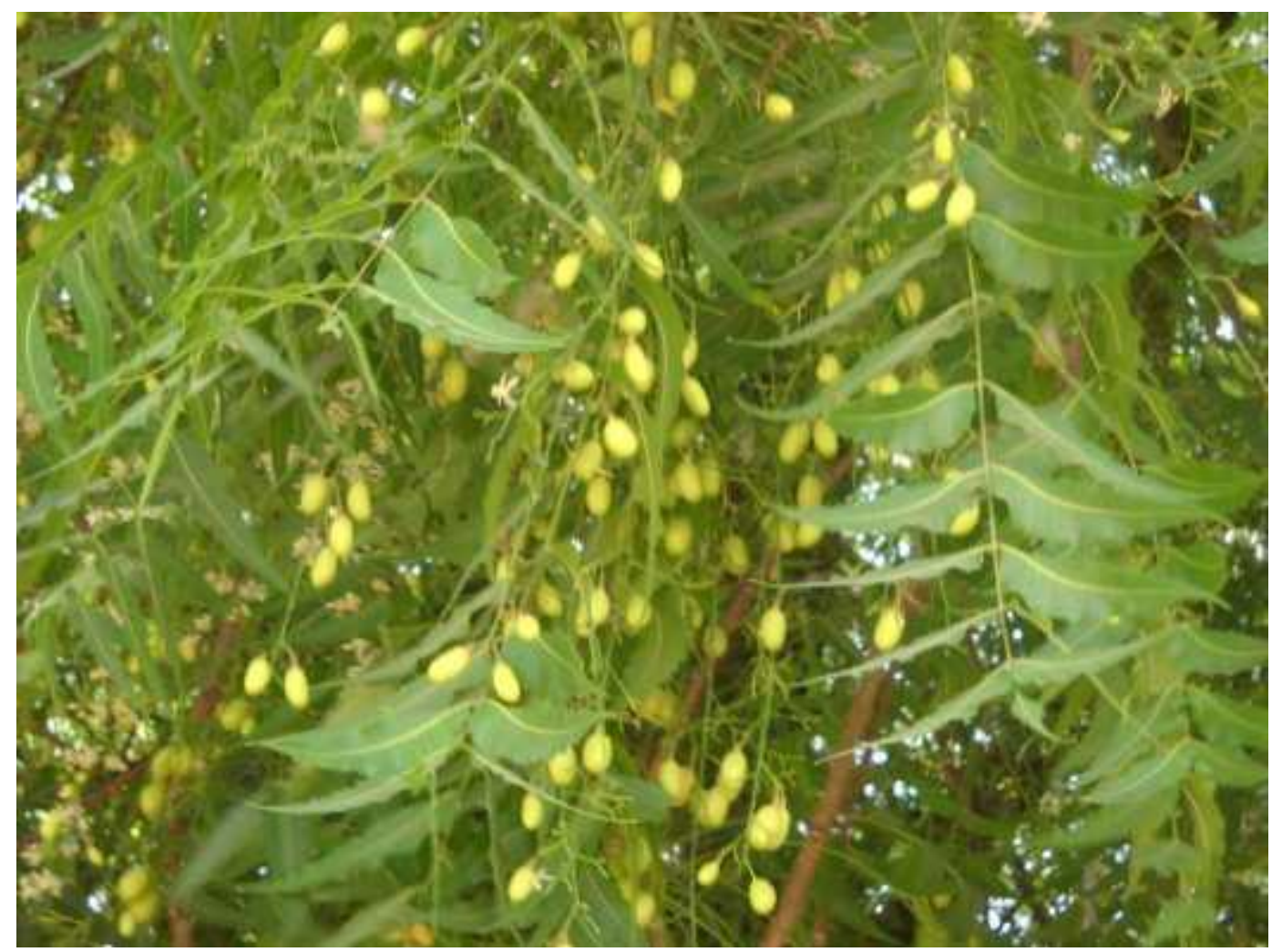

Figure 1 : Photo des fruits de neem (Source : Boubacar BASS) 


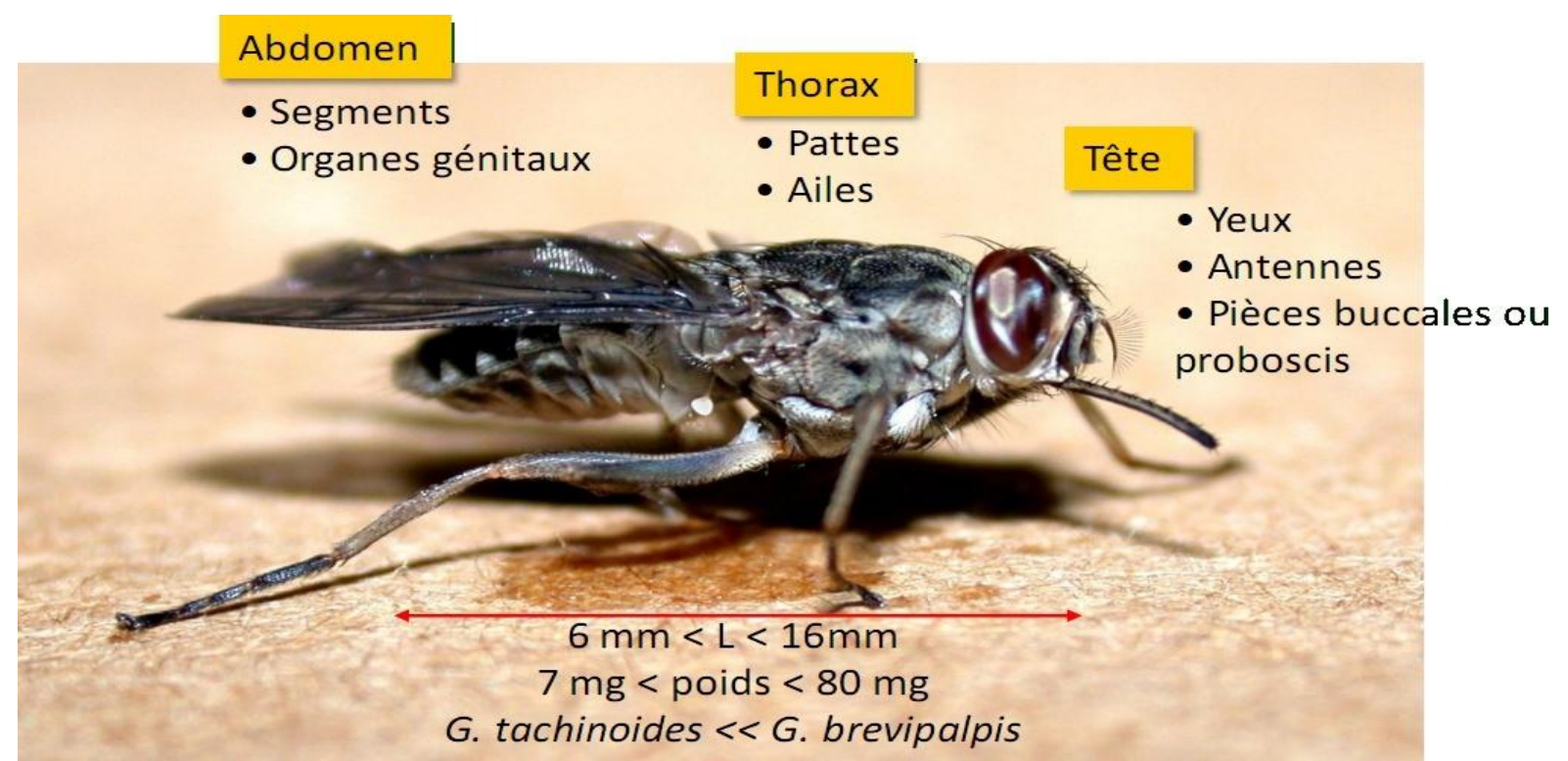

Figure 2 : Photo d'une glossine (source : Pascal Grebault) (6)

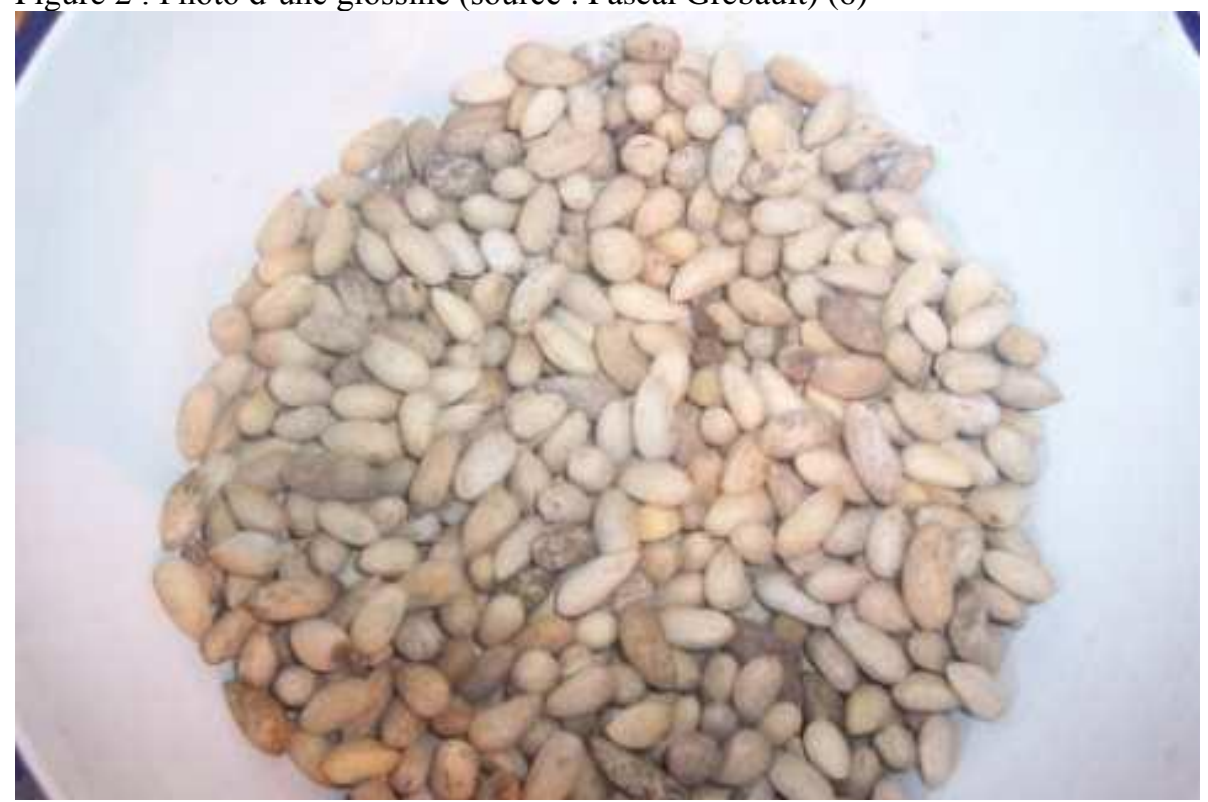

Figure 3 : Photo de graines de neem non décortiquées. (Source : Boubacar BASS)

Tableau 1: Composition centésimale en acides gras de l'huile de neem.

\begin{tabular}{lc}
\hline Acides gras & Pourcentage \\
\hline Acide Myristique C14:0 & $0,2-0,26$ \\
Acide Palmitique C16 :0 & $13,6-16,2$ \\
Acide Stéarique C18:0 & $14,4-24,1$ \\
Acide Arachidique C20 :0 & $0,8-3,4$ \\
Acide Oléique C18 :1 & $49,1-61,9$ \\
Acide Lignocérique C24:0 & - \\
Acide Linoléique C18 :2 & $2,3-15,8$ \\
\hline
\end{tabular}


Tableau 2 : Mortalité des glossines en fonction de la concentration de l'huile de neem.

\begin{tabular}{lccc}
\hline$\%$ Huile de neem & $\begin{array}{c}\text { Femelles âgées } \\
\text { et gravides } \\
(\mathrm{n}=50)\end{array}$ & $\begin{array}{c}\text { Mâles âgés de } \\
\text { plus de } 10 \text { jours } \\
(\mathrm{mn}=50)\end{array}$ & Femelles de 2-3 jours \\
\hline $2 \%$ & $1 / 50$ & $1 / 50$ & $0 / 55$ \\
$3 \%$ & $25 / 50$ & $20 / 50$ & $25 / 55$ \\
$4 \%$ & $50 / 50$ & $50 / 50$ & $55 / 55$ \\
$6 \%$ & $50 / 50$ & $50 / 50$ & $55 / 55$ \\
$7 \%$ & $50 / 50$ & $50 / 50$ & $55 / 55$ \\
$8 \%$ & $50 / 50$ & $50 / 50$ & $55 / 55$ \\
\hline
\end{tabular}

Tableau 3: Mortalité des glossines en fonction du tissu bleu imprégné avec différentes concentrations de l'huile de neem.

\begin{tabular}{llr}
\hline $\begin{array}{l}\text { Tissu bleu imprégné avec } \\
\text { différentes concentrations de } \\
\text { l'huile de neem }\end{array}$ & Femelles âgées et gravides & Mâles âgés de plus \\
\hline $2 \%$ & $0 / 50$ après $24 \mathrm{~h}$ & $0 / 50$ après $24 \mathrm{~h}$ \\
$3 \%$ & $1 / 50$ après $24 \mathrm{~h}$ & $0 / 50$ après $24 \mathrm{~h}$ \\
$4 \%$ & $5 / 50$ après $24 \mathrm{~h}$ & $3 / 50$ après $24 \mathrm{~h}$ \\
$6 \%$ & $25 / 50$ après $24 \mathrm{~h}$ & $25 / 50$ après $24 \mathrm{~h}$ \\
$7 \%$ & $50 / 50$ après $24 \mathrm{~h}$ & $50 / 50$ après $24 \mathrm{~h}$ \\
$8 \%$ & $50 / 50$ après $24 \mathrm{~h}$ & $50 / 50$ après $24 \mathrm{~h}$ \\
\hline
\end{tabular}

Tableau 4: Nombre de glossines ayant pris le repas sanguin.

\begin{tabular}{lcc}
\hline$\%$ huile de neem & $\begin{array}{c}\text { Flanc de l'animal traité avec } \\
\text { huile de neem }\end{array}$ & Flanc de l'animal non traité \\
\hline $2 \%$ & $5 / 100$ & $100 / 100$ \\
$3 \%$ & $4 / 100$ & $100 / 100$ \\
$4 \%$ & $1 / 100$ & $100 / 100$ \\
$6 \%$ & $0 / 100$ & $100 / 100$ \\
$7 \%$ & $0 / 100$ & $100 / 100$ \\
$8 \%$ & $0 / 100$ & $100 / 100$ \\
\hline
\end{tabular}


Tableau 5: Résultats des enquêtes protozoologiques chez les bovins traités avec la solution $8 \%$ huile de neem.

\begin{tabular}{lccccccccccc}
\hline Localités & Lat. & Long. & Nbre & Tbb & Tc & Tv Total & Moy. & Préval. \\
& & & & Prél. & & & Positifs & PCV \\
& & & & & & & & \\
\hline Troupeau I (Dioila) & 1249431 & 0679111 & 13 & 0 & 0 & 0 & 0 & 52 & 0 \\
Troupeau II (Dioila) & 1249616 & 0679201 & 12 & 0 & 0 & 0 & 0 & 41,7 & 0 \\
Troupeau III (Dioila) & 1249540 & 0679310 & 10 & 0 & 0 & 0 & 0 & 43,7 & 0 \\
Troupeau I (Tenebougou) & 1247941 & 0692082 & 13 & 0 & 0 & 0 & 0 & 41,2 & 0 \\
Troupeau II (Tenebougou) & 1251528 & 0678076 & 12 & 0 & 0 & 0 & 0 & 39,6 & 0 \\
Total & & 60 & 0 & 0 & 0 & 0 & 43,5 & 0 \\
\end{tabular}

Lat. : Latitude $\quad$ Long. : Longitude Nbre Prél. : Nombre de Prélèvements

Tbb: Trypanosoma brucei brucei Tc: Trypanosoma congolense Tv: Trypanosoma vivax

Moy. PCV: Moyenne du taux d'hématocrite Préval.: Prévalence

Tableau 6: Moyenne de l'hématocrite des animaux traités mensuellement et bimensuellement avec la solution $8 \%$ huile de neem.

\begin{tabular}{|c|c|c|}
\hline \multirow[t]{2}{*}{ Localités } & 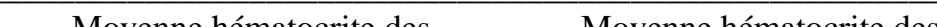 & es $\quad$ Moyenne hématocrite des \\
\hline & animaux traités par mois & animaux traités tous les 2 mois \\
\hline Troupeau I (Dioila) & 54 & 50 \\
\hline Troupeau II (Dioila) & 42,5 & 41 \\
\hline Troupeau III (Dioila) & 44,6 & 42,9 \\
\hline Troupeau I (Tenebougou) & 41,4 & 41,1 \\
\hline Troupeau II (Tenebougou) & 38,5 & 41 \\
\hline Total & 44,2 & 43,2 \\
\hline
\end{tabular}

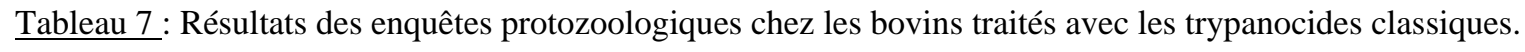

\begin{tabular}{|c|c|c|c|c|c|c|c|c|c|}
\hline Localités & Lat. & Long. & $\begin{array}{r}\mathrm{N} \\
\text { Prél. }\end{array}$ & & Tbb & $\mathrm{Tc}$ & $\begin{array}{l}\text { Tv Total } \\
\text { Positifs }\end{array}$ & ${ }^{1}{ }^{1}$ & Préval. \\
\hline Troupeau I (Dioila) & 1249431 & 0679111 & 50 & 0 & 0 & 2 & 2 & 32 & 4 \\
\hline Troupeau II (Dioila) & 1249616 & 0679201 & 50 & 0 & 1 & 3 & 4 & 28 & 8 \\
\hline Troupeau III (Dioila) & 1249540 & 0679310 & 20 & 0 & 1 & 1 & 2 & 30 & 10 \\
\hline Troupeau I (Tenebougou) & 1247941 & 0692082 & 50 & 0 & 0 & 1 & 1 & 40 & 2 \\
\hline Troupeau II (Tenebougou & 1251528 & 0678076 & 20 & 0 & 0 & 0 & 0 & 43 & 0 \\
\hline Total & & & 190 & 0 & 2 & 7 & 9 & 34,6 & 4,8 \\
\hline
\end{tabular}

Lat. : Latitude Long. : Longitude Nbre Prél. : Nombre de Prélèvements

Tbb: Trypanosoma brucei brucei Tc: Trypanosoma congolense Tv: Trypanosoma vivax

Moy. PCV: Moyenne du taux d'hématocrite Préval.: Prévalence 